\title{
Plasma ghrelin levels in association with left ventricular function and nutritional status in dialysis patients
}

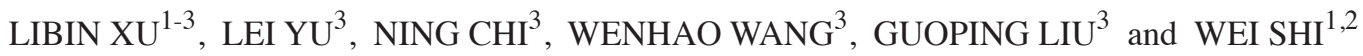 \\ ${ }^{1}$ Southern Medical University; ${ }^{2}$ Division of Nephrology, Guangdong General Hospital, Guangdong Academy of Medical \\ Sciences, Guangzhou, Guangdong 510515; ${ }^{3}$ Inner Mongolia People's Hospital, Hohhot, Inner Mongolia 010017, P.R. China
}

Received January 26, 2016; Accepted April 15, 2016

DOI: $10.3892 /$ br.2016.666

\begin{abstract}
The present study investigated the association between ghrelin levels and the cardiac function and malnutrition of dialysis patients. The aim was to examine the conducive use of exogenous ghrelin to improve the malnutrition, protect the cardiovascular function with dialysis patients in the future. The study included 30 continuous ambulatory peritoneal dialysis (CAPD) patients and 30 hemodialysis (HD) patients undertaking treatment between March 2013 and March 2014. The control group included a total of 30 healthy physical examinees. The plasma ghrelin levels were measured by the enzyme-linked immunosorbent assay to collect the clinical materials and biochemical parameters. The plasma ghrelin levels were $4.28 \pm 1.07,4.63 \pm 1.08$ and $2.00 \pm 0.48 \mathrm{ng} / \mathrm{ml}$ in the CAPD, HD and control groups, respectively, and statistical significance was identified between the three groups; $\mathrm{F}=75.106, \mathrm{P}<0.0001$. The plasma ghrelin levels in the CAPD group were positively correlated with left ventricular ejection fraction $(\mathrm{LVEF})(\mathrm{r}=0.506, \mathrm{P}=0.004)$ and were negatively correlated with body mass index $(\mathrm{BMI})(\mathrm{r}=-0.556, \mathrm{P}=0.001)$. The plasma ghrelin levels in the CAPD and HD groups were positively correlated with serum creatinine (Scr) and blood urea nitrogen (BUN). In conclusion, the plasma ghrelin levels of patients in the CAPD and HD groups were higher compared to those of the control group, which demonstrated that dialysis patients could not effectively remove the plasma ghrelin. The present study found that the plasma ghrelin levels were positively correlated with LVEF, and high levels of ghrelin will exhibit protective effects on the cardiovascular function of CAPD patients. Plasma ghrelin levels were positively correlated with Scr and BUN levels in CAPD and HD patients, and were negatively correlated with BMI in CAPD patients,
\end{abstract}

Correspondence to: Dr Wei Shi, Division of Nephrology, Guangdong General Hospital, Guangdong Academy of Medical Sciences, 106 Zhongshan Er Road, Guangzhou, Guangdong 510515, P.R. China

E-mail: nmgxulibin@sina.com

Key words: ghrelin, dialysis, left ventricular function, malnutrition which showed that ghrelin was correlated with malnutrition of dialysis patients.

\section{Introduction}

The development of dialysis technology makes the long-term survival of the end-stage renal disease patient possible. However, mortality rates for the dialysis patients remain, mainly due to malnutrition, inflammation and cardiovascular problems. According to the latest statistics, 35-50\% of the dialysis patients succumbed due to cardiovascular complications, which is 15 times greater compared to the general population. As a newly discovered growth hormone-releasing peptide, the association of ghrelin with cardiovascular disease and malnutrition in dialysis patients is receiving increased attention.

The molecular weight of ghrelin is $3.3 \mathrm{kD}$ and its particle diameter ranges $90-150 \mathrm{~nm}$. The human ghrelin gene is located on chromosome $3 \mathrm{q} 25-26$. Ghrelin mRNA is translated into the protein precursor containing 117 amino acids and following slicing becomes ghrelin containing 28 amino acids (1). Ghrelin has two types of molecular forms; symplectic acylated ghrelin (acyl ghrelin) and des-acyl ghrelin. Acyl ghrelin has an n-octanoylated serine in position 3, and this modification is essential for the activity of ghrelin (2). The majority of animal and human tests have proved that the total ghrelin level can stimulate growth hormone secretion, enhance appetite, improve feeding, adjust the positive balance of energy, regulate gastric motility, promote gastric acid secretion, protect gastric mucosa, provide direct and indirect protection for the cardiovascular system, and improve the immune system function (3).

The present study aimed to understand the association between ghrelin and cardiovascular function by measuring the plasma ghrelin levels of the hemodialysis (HD) patients, continuous ambulatory peritoneal dialysis (CAPD) patients and the control group, by analyzing the correlation between ghrelin and the associated indexes and by exploring the correlation between the plasma ghrelin levels and their corresponding echocardiography indicators in these three groups. A previous study indicates that ghrelin is closely associated with malnutrition, inflammation and atherosclerosis (4). The study of the correlation between ghrelin and malnutrition with dialysis patients has been reported previously (5), but 
the correlation between ghrelin and cardiovascular function in patients with dialysis remains to be assessed. Therefore, the association between ghrelin levels, cardiac function and malnutrition in dialysis patients remains to be further studied. The present study aimed to further clarify these associations to provide better protection for the cardiovascular function of patients by adopting ghrelin as a primary timing indicator of the nutritional intervention for the therapy of dialysis patients, which can reduce the mortality rate and improve the patient quality of life.

\section{Materials and methods}

Patients and healthy controls. A total of 30 CAPD patients were selected for the CAPD group from the Inner Mongolia People's Hospital (Hohhot, China) between March 2013 and March 2014. The CAPD time of the patient was $\geq 3$ months (19 males and 11 females, with an average age of $55.43 \pm 13.82$ years) using the peritoneal fluid produced by Guangzhou Baxter Healthcare (Guangzhou, China) and their dialysis doses were between 6-8 litres every 24 h. For the HD group, clinical data was selected from the patients who underwent the dialysis treatment with bicarbonate dialysate at the same time as the CAPD group in our blood purification center for $>3$ months. The control group consisted of 30 healthy physical examinees in our hospital during the same period who were age- and gender-matched to the CAPD group. For all three groups, the possibility was excluded of developing during the observation period severe infection, malignant tumor, blood disease, active lupus erythematosus, undergoing hormone and immunosuppressive therapy, rheumatic heart disease, cor pulmonale, congenital heart disease and hyperthyroidism heart disease. The protocol was approved by the Medical Ethics Committee of the Guangdong General Hospital, Inner Mongolia People's Hospital and the Southern Medical University. Informed written consent was obtained from all the participants prior to participation in the study.

Research methods. The subjects provided detailed clinical data, including history and laboratory examination results. The Vivid T Color Doppler Ultrasonic Diagnostic instrument produced by GE Healthcare (Pittsburg, PA, USA) was used for analysis of all groups and $2 \mathrm{ml}$ of fasting venous blood was obtained from all participants the morning after clinical treatment or hospitalization. Within $30 \mathrm{~min}$ of obtaining the sample, the ghrelin blood specimens were placed in an anticoagulant tube with an appropriate amount of ethylenediaminetetraacetic acid and were centrifuged for $15 \mathrm{~min}$ at $1,509.3 \mathrm{x}$ g. The supernatant fluid was removed and stored in the refrigerator at $-80^{\circ} \mathrm{C}$. The ghrelin enzyme-linked immunosorbent assay kit was produced by R\&D Systems, Inc. (New Brunswick, NJ, USA).

Statistical analysis. Statistical software was used to analyze the data with all the values expressed as mean \pm standard error of the mean. The independent sample t-test was adopted for the comparison of the mean of the two samples and the Pearson coefficient was used for the correlation analysis. SPSS version 13.0 software (SPSS, Inc., Chicago, IL, USA) was used
Table I. Comparison of the plasma ghrelin levels between the three groups.

\begin{tabular}{lccc}
\hline Group (n) & Ghrelin, ng/ml & F & P-value \\
\hline Hemodialysis (30) & $4.63 \pm 1.08$ & 75.106 & $<0.0001$ \\
Peritoneal dialysis (30) & $4.28 \pm 1.07$ & & \\
Control (30) & $2.00 \pm 0.48$ & & \\
\hline
\end{tabular}

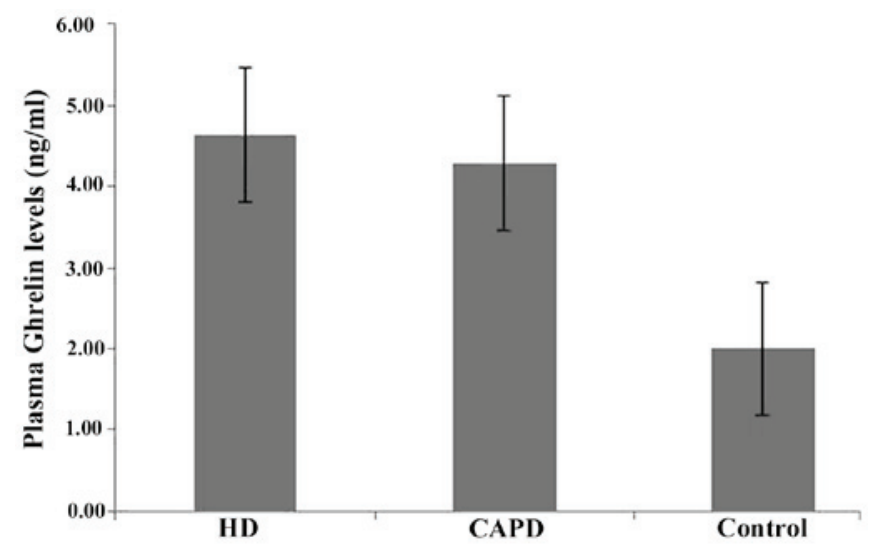

Figure 1. Plasma levels of total ghrelin $(\mathrm{ng} / \mathrm{ml})$ in patients with ambulatory peritoneal dialysis (CAPD), haemodialysis (HD) and in healthy controls. The plasma ghrelin levels of patients on CAPD group and HD group were significantly higher than those of the control group, ${ }^{*} \mathrm{P}<0.0001$.

for these analyses and $\mathrm{P}<0.05$ was considered to indicate a statistically significant difference.

\section{Results}

Comparison of plasma ghrelin levels between the three groups. Differences obtained by single-factor analysis of variance between the three groups were considered to indicate a statistically significant difference (F-value, 75.106; $\mathrm{P}<0.0001$ ), and the comparison between two groups also exhibited statistical significance $(\mathrm{P}<0.05)$. The plasma ghrelin levels of the $\mathrm{HD}$ and CAPD groups were significantly higher compared to that of the controls (Table I and Fig. 1).

Correlation analysis between the plasma ghrelin levels and echocardiography in the three groups. The plasma ghrelin levels of CAPD patients were positively correlated with left ventricular ejection fraction $(\mathrm{LVEF})(\mathrm{r}=0.506, \mathrm{P}=0.004)$ (Table II).

Correlation analysis between the plasma ghrelin levels and laboratory index in the three groups. The present study compared the plasma ghrelin levels and the association between each laboratory index, which indicates that plasma ghrelin levels showed a positive correlation with serum creatinine ( $\mathrm{Scr}$ ) (CAPD group, $\mathrm{r}=0.506$ and $\mathrm{P}=0.004$; HD group, $\mathrm{r}=0.478$ and $\mathrm{P}=0.008)$, blood urea nitrogen (BUN) (CAPD group, $\mathrm{r}=0.649$ and $\mathrm{P}=0.002$; HD group, $\mathrm{r}=0.582$ and $\mathrm{P}=0.001$ ). The present study shows that the plasma ghrelin levels of CAPD patients were negatively correlated with BMI $(\mathrm{r}=-0.556, \mathrm{P}=0.001)$ (Table III). 
Table II. Correlation analysis between plasma ghrelin levels and the echocardiographic in the three groups.

\begin{tabular}{|c|c|c|c|c|c|c|}
\hline \multirow[b]{2}{*}{ Echocardiographic } & \multicolumn{2}{|c|}{ Haemodialysis } & \multicolumn{2}{|c|}{ Peritoneal dialysis } & \multicolumn{2}{|c|}{ Control } \\
\hline & $\mathrm{r}$ & P-value & $\mathrm{r}$ & P-value & $\mathrm{r}$ & P-value \\
\hline IVST & -0.018 & 0.932 & 0.037 & 0.844 & -0.024 & 0.901 \\
\hline LVPWT & -0.041 & 0.567 & 0.142 & 0.455 & 0.060 & 0.754 \\
\hline LVEDD & 0.023 & 0.806 & -0.068 & 0.720 & 0.001 & 0.996 \\
\hline LAD & 0.185 & 0.356 & 0.080 & 0.675 & -0.018 & 0.924 \\
\hline LVM & 0.206 & 0.184 & 0.077 & 0.684 & 0.023 & 0.903 \\
\hline LVMI & 0.132 & 0.508 & -0.080 & 0.676 & 0.104 & 0.583 \\
\hline LVEF & 0.076 & 0.537 & 0.506 & $0.004^{\mathrm{a}}$ & -0.120 & 0.528 \\
\hline
\end{tabular}

${ }^{a} \mathrm{P}<0.01$, the plasma ghrelin levels in CAPD group were positively correlated with left ventricular ejection fraction. IVST, interventricular septum thickness in diastole; LVPWT, left ventricular posterior wall thickness in diastole; LVEDD, left ventricular end-diastolic dimension; LAD, left atrial diameter; LVM, left ventricular mass; LVMI, left ventricular mass index; LVEF, left ventricular ejection fraction.

Table III. Correlation analysis between plasma ghrelin levels and the laboratory index in the three groups.

\begin{tabular}{|c|c|c|c|c|c|c|}
\hline \multirow[b]{2}{*}{ Laboratory index } & \multicolumn{2}{|c|}{ Haemodialysis } & \multicolumn{2}{|c|}{ Peritoneal dialysis } & \multicolumn{2}{|c|}{ Control } \\
\hline & $\mathrm{r}$ & P-value & $\mathrm{r}$ & P-value & $\mathrm{r}$ & P-value \\
\hline $\mathrm{Scr}, \mu \mathrm{mol} / 1$ & 0.478 & $0.008^{\mathrm{a}}$ & 0.506 & $0.004^{\mathrm{a}}$ & 0.113 & 0.552 \\
\hline BUN, mmol/1 & 0.582 & $0.001^{\mathrm{a}}$ & 0.649 & $0.002^{\mathrm{a}}$ & 0.267 & 0.153 \\
\hline $\mathrm{Hb}, \mathrm{g} / \mathrm{l}$ & -0.016 & 0.933 & 0.091 & 0.633 & -0.224 & 0.235 \\
\hline $\mathrm{Ca}, \mathrm{mmol} / \mathrm{l}$ & 0.188 & 0.320 & 0.073 & 0.701 & -0.258 & 0.169 \\
\hline $\mathrm{P}, \mathrm{mmol} / \mathrm{l}$ & -0.150 & 0.429 & 0.177 & 0.351 & -0.339 & 0.067 \\
\hline PTH, pg/ml & -0.045 & 0.814 & -0.223 & 0.235 & 0.092 & 0.630 \\
\hline $\mathrm{TP}, \mathrm{g} / \mathrm{l}$ & -0.139 & 0.464 & -0.042 & 0.824 & 0.001 & 0.997 \\
\hline ALB,$g / 1$ & -0.053 & 0.780 & 0.258 & 0.168 & -0.037 & 0.845 \\
\hline $\mathrm{TG}, \mathrm{mmol} / \mathrm{l}$ & 0.173 & 0.362 & 0.035 & 0.854 & 0.062 & 0.747 \\
\hline $\mathrm{TC}, \mathrm{mmol} / \mathrm{l}$ & 0.206 & 0.274 & 0.232 & 0.218 & 0.090 & 0.636 \\
\hline HDL-L, mmol/1 & 0.042 & 0.827 & -0.018 & 0.924 & -0.339 & 0.007 \\
\hline LDL-L, mmol/l & 0.209 & 0.269 & 0.063 & 0.740 & 0.288 & 0.123 \\
\hline BMI, $\mathrm{kg} / \mathrm{m}^{2}$ & & & -0.556 & $0.001^{\mathrm{a}}$ & & \\
\hline Weight, kg & & & -0.213 & 0.259 & & \\
\hline
\end{tabular}

${ }^{a} \mathrm{P}<0.01$, the plasma ghrelin levels in CAPD groupand HD group was positively correlation with serum creatinine (Scr) and urea nitrogen (BUN). The plasma ghrelin levels in CAPD group were significantly negative correlated with body mass index (BMI). Hb, hemoglobin; Ca, calcium; PTH, parathyroid hormone; TP, total protein; ALB, albumin; TG, triglyceride; TC, total cholesterol; HDL, high-density lipoprotein; LDL, low-density lipoprotein.

\section{Discussion}

Animal experiments and clinical trials have confirmed that ghrelin is significant in promoting growth hormone-releasing material, such as the growth hormone secretagogue receptor (GHSR), and has a dose-effect correlation with growth hormone secretion. It can also stimulate more growth hormone secretion compared with dose GHSR and unnatural GHS (6). First ghrelin can enhance appetite, increase energy intake and maintain a positive balance of energy. Therefore, ghrelin can improve malnutrition of dialysis patients, and additionally, ghrelin can enhance myocardial contraction force, increase LVEF significantly, inhibit vascular calcification, increase the coronary circulation, improve ischemia reperfusion, and have a direct or indirect protection on the cardiovascular system. Therefore, the present study aimed to analyze, using the plasma ghrelin levels in CAPD, HD and healthy controls, the plasma ghrelin level and correlation of each index in patients, and the association between plasma ghrelin levels and each group echocardiography. The study also aimed to further define the plasma ghrelin levels, cardiovascular function and malnutrition in patients with dialysis, assess the conducive 
use of exogenous ghrelin to improve malnutrition and protect cardiovascular function in dialysis patients in the future, and provide novel information to improve the quality of survival and long-term survival in dialysis patients.

The majority of the studies have reported that the plasma ghrelin levels in dialysis patients were significantly higher compared to the age- and gender-matched control group (7). The results of the present study indicate a significantly greater increase in ghrelin levels in the HD and CAPD groups compared to the control group, which is consistent with the conclusions of the majority of recent studies. Pérez-Fontán et al (8), following the investigation of plasma ghrelin levels in age-matched control groups, reported that the plasma ghrelin levels of 20 HD patients and 21 peritoneal dialysis patients were significantly higher than that of the control group; however, no significant differences were identified between the former two. Tentolouris et al (9) studied the ghrelin levels of 57 healthy controls and 122 chronic renal insufficient patients (65 HD patients and 57 non-dialysis patients). The results showed that the fasting total ghrelin plasma levels of the HD group and non-dialysis patients increased higher in comparison to the control group, and there was no evident difference between the dialysis and non-dialysis groups. There are different view points regarding the reasons for the plasma ghrelin level elevation. For example, the kidney may be the site where the clearance and degradation of the hormone occurs. A study conducted by Yoshimoto et al (10) showed that compared with the normal controls, ghrelin levels of dialysis patients increased 2.8-fold. The study also found that ghrelin levels in rats with partial nephrectomy increased by 1.3 -fold, and 3.1-fold in rats with double renal resection. Previous studies have found that ghrelin can be detected in the urine of healthy subjects, which suggests that the kidney is an important metabolic site for ghrelin. The metabolic pathway of ghrelin remains to be elucidated; however, Mori et al (11) reported that ghrelin and its receptors exist in the kidney, which means that the kidney is not only the source of ghrelin, but also its target organ. Another study suggested that the increase of total plasma ghrelin levels in patients with chronic kidney disease (CKD) is mainly caused by less removal and degradation of ghrelin by kidney, which could be one of the main reasons for the significant increase of the plasma ghrelin levels in patients with CKD. Certain investigators have suggested that the adaptive compensatory reaction in the body under the condition of the inadequate energy intake is another cause of the rising plasma ghrelin level $(8,12)$. In addition, others consider that insulin can reduce the plasma ghrelin levels in the normal population; however, it is common for non-diabetic dialysis patients to develop insulin resistance. Therefore, the weakened inhibition of plasma ghrelin levels caused by insulin resistance is also a reason for the elevated plasma ghrelin levels (13).

Cardiovascular disease is one of the major causes of mortality for dialysis patients. The incidence rate of cardiovascular disease in patients with CKD is 10-30 times greater than that of the general population. The early symptom of cardiac function insufficiency is lower left ventricular diastolic function. Left ventricular hypertrophy $(\mathrm{LVH})$ is the most common cardiomyopathy in patients with CKD, which is evident in patients with mild renal impairment (the incidence of $\mathrm{LVH}$ is $\sim 30 \%$ in CKD patients when creatinine clearance is $50-70 \mathrm{ml} / \mathrm{min}$ ), and the incidence rate increases (14) with worsened renal function damage. Renal damage leads to cardiovascular disease, which can aggravate kidney damage. This interaction forms a vicious cycle. Cardiovascular disease in patients with CKD occurs early without clear symptoms, which makes it easily ignored. Therefore, the damage is difficult to reverse once the symptom appears. The study results show that left ventricular myocardial mass index (LVMI) was $151.56 \pm 50.56 \mathrm{~g} / \mathrm{m}^{2}$ in the HD group, $157.09 \pm 46.14 \mathrm{~g} / \mathrm{m}^{2}$ in the CAPD group, and $77.27 \pm 18.34 \mathrm{~g} / \mathrm{m}^{2}$ in the control group (data not shown). Compared with the healthy controls, the LVMI levels in the HD and CAPD groups increased significantly, which indicate that $\mathrm{LVH}$ is widespread among the dialysis patients. This finding is similar to the result of a Canadian echocardiographic survey of 432 patients with CKD who just started dialysis (15). In addition, the study found that plasma ghrelin levels in CAPD patients were positively correlated with LVEF ( $r=0.506, P=0.004)$, which suggests that the higher the plasma ghrelin level reaches, the higher the LVEF scores. This may attribute to the protective effects of plasma ghrelin on cardiovascular function. No such correlation with LVEF was observed in the HD group. The increasing plasma ghrelin levels in CAPD patients also prompted LVEF, increased cardiac output, increased the end pressure of the left ventricular systolic function and increased the myocardial contraction ratio. HD patients have greater hemodynamic fluctuations, which could undermine the protective effects of ghrelin on the cardiovascular system. The present study found that LVEF was positively correlated with plasma ghrelin levels in CAPD patients, which is not consistent with the result of a study performed in Greece (8) that reported no correlation between the plasma ghrelin levels and LVEF in patients with end-stage CKD. In addition, this study showed that there was no significant correlation between the interventricular septum thickness and LVMI of CAPD patients with the plasma ghrelin levels.

Protein malnutrition is common among the dialysis patients, which can be caused by anorexia, nausea and vomiting. This leads to lack of energy and protein intake. According to previously reported statistics, every day an abdominal peritoneal dialysis patient loses, through peritoneal fluid, 8-9 $\mathrm{g}$ of protein and $2.4 \mathrm{~g}$ of amino acid. Those who develop peritonitis would lose $15 \mathrm{~g}$ of protein (16) and 10-30 g of amino acids and peptides after 1 HD. When the endocrine disorder caused by the metabolic acidosis occurs, branched chain amino acids can inhibit protein synthesis and promote the decomposition of protein, leading to a negative nitrogen balance. Peritoneal dialysis patients frequently suffer from micro-inflammatory conditions due to factors such as oxidative stress, toxin retention and biological incompatibility. Studies have shown that injections of growth hormone can evidently improve the nutritional status of the dialysis patients and reduce their risk of developing cardiovascular disease (17). Following the measurement of 41 patients with CKD in the study by Yoshimoto et al (10), the plasma total ghrelin levels were positively correlated with Scr and BUN levels, and negatively correlated with the creatinine clearance rate. However, a study performed in Taiwan suggested that the plasma ghrelin levels were significantly negatively correlated with BMI in dialysis patients, and therefore, ghrelin resistance may exist 
among dialysis patients, which was closely associated with the nutritional index (18). The present study suggests that plasma ghrelin levels were significantly positively correlated with Scr and BUN, indicating that the kidney was the important site of metabolism of ghrelin. The plasma ghrelin levels of CAPD patients were significantly negatively correlated with BMI, but no correlation was observed for BMI in HD patients, indicating that the plasma levels of ghrelin were closely associated with malnutrition in CAPD patients. This may be associated with the two types of dialysis in a different way. HD on toxin removal is intermittent and HD for the removal of the ghrelin is intermittent as ghrelin has a weak fluctuation in the body. However, toxin removal by CAPD is continuous and smooth, sustaining high levels of ghrelin, which will have a lasting impact on the nutritional status of CAPD patients. The plasma ghrelin levels from different dialysis methods indicate that the nutritional status of patients has different effects. In addition, malnutrition, inflammation and atherosclerosis are inseparable interactions in dialysis patients, and therefore, the effect of ghrelin should be noted with regards to malnutrition, inflammation and atherosclerosis, and also the occurrence and development of cardiovascular disease in dialysis patients. The aforementioned correlation analysis found that plasma ghrelin levels are associated with malnutrition in CAPD patients; however, the specific mechanism remains to be elucidated, and further investigations are required regarding the association between the plasma ghrelin levels in dialysis patients and inflammation and atherosclerosis.

In conclusion, the plasma ghrelin levels may be associated with protein malnutrition, oxidative stress and the occurrence and development of cardiovascular disease in end-stage renal disease and dialysis patients. The significant increase of ghrelin levels in HD and CAPD patients may be attributed to the declining function of the kidney to remove and metabolically degrade the hormone. However, the malnutrition and endocrine disorders of patients should be considered as another, and possibly more important, cause for this symptom. The positive correlation between the plasma ghrelin level and LVEF in CAPD patients demonstrates a significant correlation. Plasma ghrelin is a recently identified brain-gut peptide, which can increase appetite, improve energy intake, regulate weight, protect against malnutrition, protect the cardiovascular function and adjust immune system function. Therefore, further study of the cause behind the increase in plasma ghrelin levels in patients with CKD will be beneficial for the adoption of exogenous ghrelin for improving malnutrition, microinflammation and atherosclerosis in end-stage renal disease and dialysis patients, which will protect their cardiovascular function. The present study provides a novel perspective for improving the quality of life in patients and reducing complications.

\section{Acknowledgements}

The authors would like to thank the patients and healthy controls who participated in the study. The present study was funded by the Talents Fund of Inner Mongolia Autonomous Region (no. 2008).

\section{References}

1. Seim I, Collet C, Herington AC and Chopin LK: Revised genomic structure of the human ghrelin gene and identification of novel exons, alternative splice variants and natural antisense transcripts. BMC Genomics 8: 298, 2007.

2. Kojima M and Kangawa K: Structure and function of ghrelin. Results Probl Cell Differ 46: 89-115, 2008.

3. Kojima M and Kangawa K: Ghrelin: Structure and function. Physiol Rev 85: 495-522, 2005.

4. Hubacek JA, Bloudícková S, Bohuslavová R, Táborský P, Polakovic V, Sazamová M, Svítilová E, Vlasák J, Sojková I, Ryba M, et al: Ghrelin variants influence development of body mass index and plasma levels of total cholesterol in dialyzed patients. Clin Chem Lab Med 45: 1121-1123, 2007.

5. Ruperto M, Sánchez-Muniz FJ and Barril G: A clinical approach to the nutritional care process in protein-energy wasting hemodialysis patients. Nutr Hosp 29: 735-750, 2014 (In Spanish).

6. Nagaya N, Kojima M, Uematsu M, Yamagishi M, Hosoda H, Oya H, Hayashi $\mathrm{Y}$ and Kangawa K: Hemodynamic and hormonal effects of human ghrelin in healthy volunteers. Am J Physiol Regul Integr Comp Physiol 280: R1483-R1487, 2001.

7. Caliskan Y, Gorgulu N, Yelken B, Yazici H, Oflaz H, Elitok A, Turkmen A, Bozfakioglu S and Sever MS: Plasma ghrelin levels are associated with coronary microvascular and endothelial dysfunction in peritoneal dialysis patients. Ren Fail 31: 807-813, 2009.

8. Pérez-Fontán M, Cordido F, Rodríguez-Carmona A, Peteiro J, García-Naveiro R and García-Buela J: Plasma ghrelin levels in patients undergoing haemodialysis and peritoneal dialysis. Nephrol Dial Transplant 19: 2095-2100, 2004.

9. Tentolouris N, Makrilakis K, Doulgerakis D, Moyssakis I, Kokkinos A, Kyriaki D, Georgoulias C, Stathakis C and Katsilambros N: Increased plasma ghrelin levels in chronic renal failure are not associated with hemodynamic parameters. Horm Metab Res 37: 646-652, 2005.

10. Yoshimoto A, Mori K, Sugawara A, Mukoyama M, Yahata K, Suganami T, Takaya K, Hosoda H, Kojima M, Kangawa K, et al: Plasma ghrelin and desacyl ghrelin concentrations in renal failure. J Am Soc Nephrol 13: 2748-2752, 2002.

11. Mori K, Yoshimoto A, Takaya K, Hosoda K, Ariyasu H, Yahata K, Mukoyama M, Sugawara A, Hosoda H, Kojima M, et al: Kidney produces a novel acylated peptide, ghrelin. FEBS Lett 486: 213-216, 2000

12. Barazzoni R, Zanetti M, Biolo G and Guarnieri G: Metabolic effects of ghrelin and its potential implications in uremia. J Ren Nutr 15: 111-115, 2005.

13. Barazzoni R, Zanetti M, Stulle M, Mucci MP, Pirulli A, Dore F, Panzetta G, Vasile A, Biolo G and Guarnieri G: Higher total ghrelin levels are associated with higher insulin-mediated glucose disposal in non-diabetic maintenance hemodialysis patients. Clin Nutr 27: 142-149, 2008.

14. Kobayashi S, Oka M, Maesato K, Ikee R, Mano T, Hidekazu M and Ohtake T: Coronary artery calcification, ADMA, and insulin resistance in CKD patients. Clin J Am Soc Nephrol 3: 1289-1295, 2008.

15. Parfrey PS, Foley RN, Harnett JD, Kent GM, Murray D and Barre PE: Outcome and risk factors of ischemic heart disease in chronic uremia. Kidney Int 49: 1428-1434, 1996.

16. Mitsnefes MM: Cardiovascular complications of pediatric chronic kidney disease. Pediatr Nephrol 23: 27-39, 2008.

17. Feldt-Rasmussen B, Lange M, Sulowicz W, Gafter U, Lai KN, Wiedemann J, Christiansen JS and El Nahas M; APCD Study Group: Growth hormone treatment during hemodialysis in a randomized trial improves nutrition, quality of life and cardiovascular risk. J Am Soc Nephrol 18: 2161-2171, 2007.

18. Chang CC, Hung CH, Yen CS, Hwang KL and Lin CY: The relationship of plasma ghrelin level to energy regulation, feeding and left ventricular function in non-diabetic haemodialysis patients. Nephrol Dial Transplant 20: 2172-2177, 2005. 